\section{Importance of dermoscopy in diagnosis of tinea capitis: An evidence-based case report}

\author{
Aninda Marina, Marsha Bianti, \\ Sandra Widaty, Eliza Miranda \\ Department of Dermatology and \\ Venereology, Faculty of Medicine \\ Universitas Indonesia/Dr. Cipto \\ Mangunkusumo National General \\ Hospital, Jakarta, Indonesia
}

\begin{abstract}
Diagnosis of tinea capitis requires direct microscopy with potassium hydroxide $(\mathrm{KOH})$ staining and culture examination as the gold standard. Dermoscopy for the diagnosis of tinea capitis needs to be appraised through evidence-based case report method. A 22 years old woman came with systemic lupus erythematosus which was treated with methylprednisolone and azathioprine for a year. She had wide patchy alopecia, dry scaling, dull gray hair and minimal inflammation. KOH 20\% showed septate hyphae and spores surrounding her hair shafts. Dermoscopy examination showed "comma hairs", "corkscrew", "perifollicular scaling" and "broken hairs". Three articles from Pubmed and Gale fulfilled the inclusion and exclusion criteria. Articles were critically appraised and concluded that comma hairs sensitivity rate varies between $60-66 \%$ and specificity of $100 \%$, while corkscrew had sensitivity rate varies $20-80 \%$ and specificity of $83-100 \%$. Combination of dermoscopy with clinical performance and direct microscopic examinations amplify definitive diagnosis. Further investigations are needed to validate the role of dermoscopy as a diagnostic tool per se for tinea capitis.
\end{abstract}

\section{Introduction}

Tinea capitis (TC) is a fungal infection of the scalp that can be dry which accounts for majority of the cases $(90 \%)$ or the acute inflammatory oozing form. Diagnosis can be conducted easily by direct $10 \%-20 \%$ potassium hydroxide examination of plucked hair, or by isolation of the dermatophyte in Sabouraud agar which would take weeks to reveal and delays diagnosis and initiation of a proper treatment. TC has diverse causative agents and can exhibit with one or multiple foci of alopecia. The readings of a specific dermoscopic finding can lead to a straightforward diagnosis. ${ }^{1,2}$

Recently, the dermoscopic examination of the hair and scalp (trichoscopy) has rou- tinely done and proven to be a very effective, and efficient tool for screening and to establish the diagnosis of some hair disorders; for instance, infectious diseases such as TC. The presence of certain trichoscopic features allows speedy identifying and differentiating between these entities. The most commonly reported trichoscopic features in TC patients have been coma hairs and corkscrew hairs (CHs), which have been described as markers of TC. ${ }^{2-5}$ Trichoscope is considered the stethoscope of the dermatologist. Trichoscopy (hair and scalp dermoscopy) may be performed with a handheld dermoscope. This makes it a modern, non-invasive technique, which is well accepted by both, dermatologists and patients. Thus, trichoscopic examination of hair and scalp became an essential method in evaluation of patients with hair loss. ${ }^{6}$

\section{Clinical Question}

Can dermoscopy replace culture for diagnosing tinea capitis? How much sensitivity and specificity dermoscopy examination in diagnosing tinea capitis?

$\mathrm{P}$ : patients with suspected tinea capitis

I : dermoscopy examination

$\mathrm{C}:$ culture

$\mathrm{O}$ : Diagnosis tinea capitis

Clinical question type: diagnosis

\section{Case Report}

22-year-old woman came to the clinic, presented with an intense itchy, painless scaly patches of hair loss in the occipital region of the scalp since the past year. Complaints of scratchy or scaly plaques on the hands, feet, or other body parts are absent. She has a long-hair cat as a pet. There were no similar complaints on the family. She never did any gardening nor exchanging clothes with other people. She has systemic lupus erythematosus disease which treated with methylprednisolone and azathioprine for almost a year. She was misdiagnosed by internist whom mistaken her hair loss due to the systemic lupus erythematosus itself rather than other disease or infection. On physical examination, there are existences of wide patchy alopecia on occipital region of the scalp with massive dry scaling, dull gray hair and minimal inflammation. There were no palpable lymph nodes in the lateral cervical chains. Potassium hydroxide $20 \%$ examination showed septate hyphae and spores surrounding hair shafts (ectothrix). The Woods lamp examination yielded a characteristic fluorescence of yellowish green.

Portable dermoscopy examination at 10x magnification (low and high magnifica-
Correspondence: Aninda Marina, Faculty of Medicine Universitas Indonesia/Dr. Cipto Mangunkusumo National General Hospital, Jakarta. J1. Salemba Raya, No. VI, RW.5, Kenari, Senen, Central Jakarta, Jakarta 10430, Indonesia.

Tel.: +62-81386282470

E-mail: aninda.marina@gmail.com

Key words: dermoscopy, trichoscopy, tinea capitis, sensitivity, specificity

Contributions: the authors contributed equally. Conflict of interest: the authors declare no potential conflict of interest

Funding sources: None

Received for publication: 1 February 2019. Accepted for publication: 20 February 2019.

This work is licensed under a Creative Commons Attribution-NonCommercial 4.0 International License (CC BY-NC 4.0).

CCopyright A. Marina et al., 2019

Licensee PAGEPress, Italy

Dermatology Reports 2019; 11(s1):8072

doi:10.4081/dr.2019.8072

tion) was employed with alcohol as the interface medium and the most characteristic findings observed were multiple broken hairs, perifollicular scaling and hairs with a characteristic commalike shape which is called as comma hairs, and some corkscrew hairs. Since culture as the gold standard examination for tinea capitis (TC) could not be done because of the expensive cost, the doctor wondering if it possible to establish diagnosis by dermoscopy (Figure 1).

Literature search through Pubmed, Gale and Cochrane was done on January 14th 2018 using keywords 'dermoscopy' OR 'trichoscopy' AND ‘tinea capitis'. Inclusion criteria were articles in English, less than 10 years and diagnostic studies. Exclusion criteria were review articles or case report. Articles obtained then filtered with inclusion and exclusion criteria so that the resulting article is expected to answer the formulation of the problem. Fifty-four articles were obtained from literature searching. First selection was based on duplicate screening then title/abstract screening, with elimination for same articles. There was no journal found in Cochrane. The remaining were re-assessed based on inclusion criteria. Three articles were suitable for our EBCR clinical question and were critically appraised using CEBM critical appraisal worksheet. (www.cebm.net/criticalappraisal/) (Figure 2). Level of evidence was evaluated using Oxford Centre for Evidence-based Medicine criteria which 


\section{Case Report}

best to worst are 1 to 5 . Three articles were obtained from literature. Critical Appraisal Three relevant studies by Amer, et al., Brasileiro, et al., and Ekiz, et al. were critically appraised for their validity, importance, and applicability. It is important to note that all the studies lacked validity. All the clinicians were not blind to the examination. All the studies have level of evidence 4 which caused by case control methods that have low level power for diagnostic studies (Table 1). Ekiz et al., showed Corkscrew have $80 \%$ sensitivity and $100 \%$ specificity but yet Amer et al. and Brasileiro et al. showed very low sensitivity (20\%$26 \%$ ). Comma hair was found less sensitive in dermoscopy pattern compare to Corkscrew (60\%). Since all the studies were case control so there was no blinding that could result a bias in specificity (Table 2). Applicability were appraised from diagnostic sheet which the question is "Were the methods for performing the test described in sufficient detail to permit replication?". We would answer yes to Amer et $a l^{7}$ and Ekiz et al. ${ }^{10}$ studies but both needs a bigger sample size and also blinding so there would be no bias. Better methods to use would be individual cross-sectional studies with consistently applied reference standard and blinding. The best method is a systematic review of cross sectional studies with consistently applied reference standard and blinding. Yet we would answer no to Brasileiro's et al. ${ }^{8}$ study since the sensitivity is too low for diagnostic. In Amer study, ${ }^{7} 60$ subjects were divided into two study groups which 40 are diagnosed with TC and 20 diagnosed with other disease such as alopecia

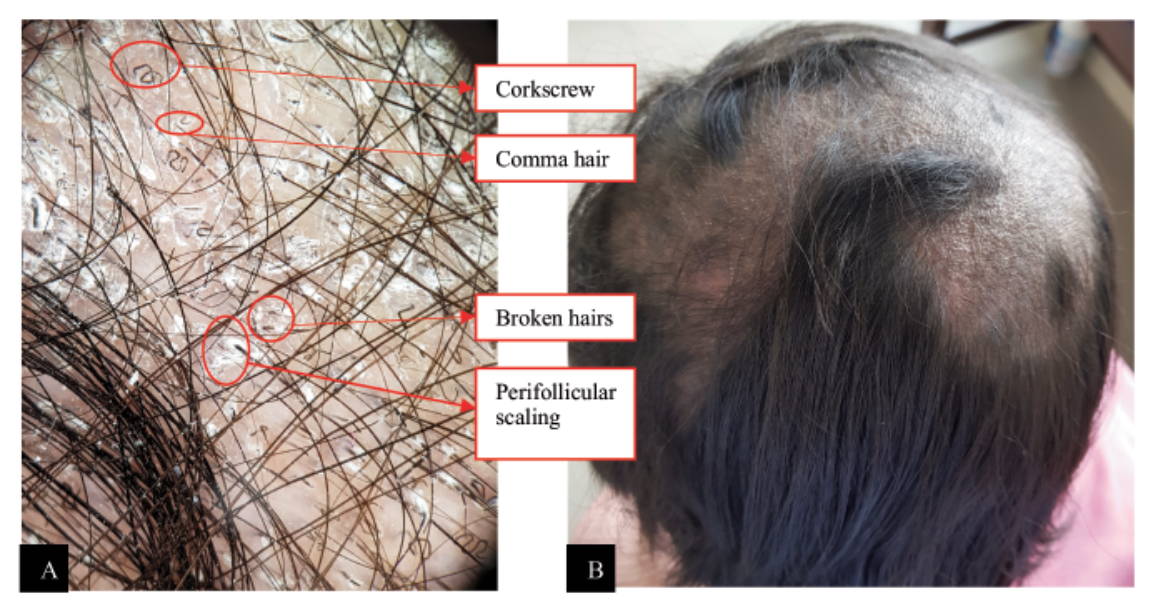

Figure 1. A) Dermoscopy from tinea capitis. B) Clinical appearance: wide patchy alopecia.

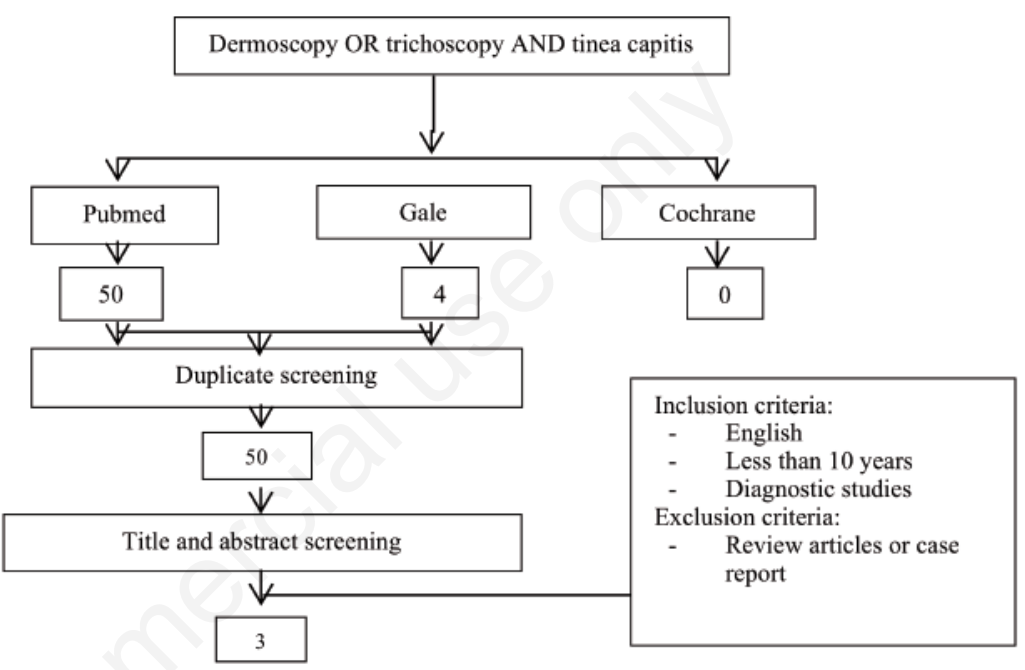

Figure 2. Literature searching strategy.

Table 1. Validity and Level of Evidence.

\begin{tabular}{|c|c|c|c|c|c|c|}
\hline Article & $\begin{array}{l}\text { Sample } \\
\text { size }\end{array}$ & $\begin{array}{l}\text { Patient } \\
\text { spectrum }\end{array}$ & $\begin{array}{l}\text { Validity* } \\
\text { Reference } \\
\text { standart }\end{array}$ & Blinding & $\begin{array}{l}\text { Gold-standart } \\
\text { comparison }\end{array}$ & $\begin{array}{c}\text { Level of } \\
\text { evidence(LoE)** }\end{array}$ \\
\hline Amer, et al. ${ }^{7}$ & 60 & + & + & - & + & 4 \\
\hline Brasileiro, et al. ${ }^{8}$ & 50 & + & + & - & + & 4 \\
\hline Ekiz, et al. ${ }^{9}$ & 25 & + & + & - & + & 4 \\
\hline
\end{tabular}

${ }^{*}+$ : done in research, $-:$ not done, ${ }^{* *}$ Level 4: Case-control studies, or "poor or non-independent reference standard**

Table 2. Sensitivity and Specificity of Dermoscopy in Diagnosing Tinea Capitis (Importance).

\begin{tabular}{|c|c|c|c|}
\hline \multirow[t]{2}{*}{ Article } & \multicolumn{2}{|c|}{ Dermoscopy Results } & \multirow[t]{2}{*}{ *Additional } \\
\hline & Sensitivity & Specificity & \\
\hline Amer, et al..$^{7}$ & $\begin{array}{l}\text { Comma hairs: } 60 \% \\
\text { Corkscrew: } 20 \%\end{array}$ & $\begin{array}{l}\text { Comma hairs: } 100 \% \\
\text { Corkscrew: } 100 \%\end{array}$ & $\begin{array}{ll}\text { Comma hair } & \text { NPV : } 55 \% \\
\text { Corkscrew } & \text { NPV : } 38 \%\end{array}$ \\
\hline Brasileiro, et al. ${ }^{8}$ & $\begin{array}{l}\text { Comma hairs: } 13 \% \\
\text { Corkscrew: } 26 \%\end{array}$ & $\begin{array}{l}\text { Comma hairs: } 83 \% \\
\text { Corkscrew: } 83 \%\end{array}$ & 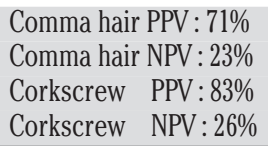 \\
\hline Ekiz, et al. ${ }^{9}$ & $\begin{array}{l}\text { Comma hairs: } 66 \% \\
\text { Corkscrew: } 80 \%\end{array}$ & $\begin{array}{l}\text { Comma hairs: } 100 \% \\
\text { Corkscrew: } 100 \%\end{array}$ & $\begin{array}{ll}\text { Comma hair } & \text { NPV : } 67 \% \\
\text { Corkscrew } & \text { NPV : } 77 \%\end{array}$ \\
\hline
\end{tabular}

*Positive predicted value (PPV) \& Negative predicted value (NPV) 
areata (AA). The selected patients had patchy hair loss, were diagnosed clinically as TC or AA without sex predilection, aged 2-12 years, and had solitary or multiple lesions of patchy hair loss of the scalp. Microscopic examination consisted of skin scraping and plucked hairs. Dermoscopic examination was carried out using portable dermoscopy at 1040 -fold magnification. The most common trichoscopic features of TC in this study were comma hairs seen in $24(60 \%)$ patients, followed by zigzag hairs seen in $12(30 \%)$, then black dots in $12(30 \%)$, Morse code hairs seen in $11(27.5 \%)$, corkscrew hairs in 8 $(20 \%)$, short broken hairs in $7(17.5 \%)$, and "I" hairs were found in $2(5 \%)$. Brasileiro et al., ${ }^{8}$ included 50 patients, of which mycological examination was positive in 38 patients (group A) and negative in 12 (group B). No significant differences concerning sex, age or race distribution between the groups were found. Mycologically negative (group B) patients were followed up and eventually diagnosed with other conditions such as alopecia areata, traction alopecia and atopic dermatitis. The most common findings were perifollicular scaling, diffuse scaling and broken hairs, observed in the majority of the patients with TC. On the other hand, black dots, corkscrew hairs, pustules and comma hairs were seen in about one-third or fewer of those patients. The frequency of each trichoscopic finding did not differ significantly between the groups $(\mathrm{P}>0.05)$, meaning that not one of these features alone could have been a predictor of TC. In Ekiz study, ${ }^{9} 25$ subjects were divided into two study groups such as 15 subjects with TC and 10 subjects diagnosed with AA clinically as a control group. Fungal cultures were performed with Saboraud dextrose. In all cases, the affected areas of the scalp were examined by a digital dermoscopy. In the trichoscopic examination, broken and dystrophic hairs $(100 \%)$ were observed on all patients with TC. Corkscrew hairs $(80 \%)$, comma hairs $(66.7 \%)$ and black dots $(13.3 \%)$ were observed respectively.

\section{Discussion}

Publication of studies involving diagnosing tinea capitis through dermoscopy in cross-sectional design were apparently lacking, therefore any conclusion of superior sensitivity or specificity of dermoscopy examination over gold standart can't be over emphasized. All of the selected for this paper were case-control studies and neither were blinded. Blinding could not be done because the examiner needs to be sure of the diagnosis first and still exploring the specific characteristic in dermoscopy. This could lead to spectrum bias in the results. Two of the stud- ies have comparable sample size, ranging from 50 to 60 subjects while Ekiz et al. only have 25 subjects. This number was too small for a diagnostic and might not represent the target population or prevalence. The small sample size in both groups and group asymmetry limited the power of statistical analysis. ${ }^{10}$ Brasiliero et al. has the lowest sensitivity for comma hair (13\%) that controvert with other two studies (Ekiz et al. and Amer et al.) or previous such as Slowinska et al. whom described the presence of comma hairs in two patients with TC and suggested them as specific markers for the condition. ${ }^{4}$ Comma hair is believed as a result of subsequent cracking and bending of a hair shaft filled with hyphae. This association has been further corroborated by other isolated cases and small series of patients with TC.,2,311 Additionally two subjects from Brasiliero study showed repeatedly negative mycological examinations and no evidence of disease progression in the absence of antifungal treatment. Those challenges the concept that comma hairs are a specific dermoscopic marker for TC. Yet all the three studies showed perifollicular scaling and broken hairs reach $100 \%$. Nevertheless broken hairs are also found in alopecia areata., ${ }^{2,9}$ Regarding corkscrew hairs, these have also been described as specific findings for TC. ${ }^{2,5,12}$ Ekiz et al. found corkscrew sensitivity up to $80 \%$. This data contradicted the other two studies as none of the studied dermoscopy findings alone proved to be specific for the diagnosis of TC. In contrast, the presence of both comma hairs or corkscrew or perifollicular scaling or dystrophic hair or broken hairs correlated with a positive mycological examination, thus suggesting this association as a more reliable indicator of TC in clinical practice. While fungal culture remains the gold standard in TC diagnosis, but we still consider dermoscopy as valuable additional tool that fast, efficient and could lead to establish the diagnosis. Further investigations are needed to validate the role of dermoscopy as a diagnostic tool per se for $\mathrm{TC}$ because the level of evidence is still low from available studies and made it inconclusive. It can be an additional tool to help the dermatologist in the diagnosis of this disorder, but always with the combination of clinical performance and mycological examination to enable definitive diagnosis.

\section{Conclusions}

Dermoscopy can be used as a fast, inexpensive and non-invasive additional diagnostic tool to differentiate tinea capitis with other alopecia. Mycological examination remains the gold standard in diagnosis of tinea capitis. Combination of dermoscopy examination such as comma hairs or corkscrew or perifollicular scaling or dystrophic hair or broken hairs with clinical performance and direct microscopy examination amplify definitive diagnosis.

\section{References}

1. Hay RJ. Tinea Capitis: Current Status. Mycopathologia. 2017;182(1-2):87-93.

2. Pinheiro AMC, Lobato LA, Varella TCN. Dermoscopy findings in tinea capitis: case report and literature review. An Bras Dermatol. 2012;87(2): 313-4.

3. Mapelli ETM, Gualandri L, Cerri A, Menni S. Comma Hairs in Tinea Capitis: A Useful Dermatoscopic Sign for Diagnosis of Tinea Capitis. Pediatr Dermatol. 2012;29(2):223-4.

4. Slowinska M, Rudnicka L, Schwartz RA, Kowalska-Oledzka E, Rakowska A, Sicinska J, et al. Comma hairs: A dermatoscopic marker for tinea capitis: A rapid diagnostic method. J Am Acad Dermatol. 2008;59(5):77-9.

5. Elghblawi E. Idiosyncratic findings in trichoscopy of tinea capitis: Comma, zigzag hairs, corkscrew, and morse code-like hair. Int J Trichology. 2016;8(4):180.

6. Rudnicka L, Olszewska M, Rakowska A, Slowinska M. Trichoscopy update 2011. J Dermatol Case Rep. 2011;5(4): 82-8.

7. Amer M, Helmy A, Amer A. Trichoscopy as a useful method to differentiate tinea capitis from alopecia areata in children at Zagazig University Hospitals. Int J Dermatol. 2017;56(1): 116-20.

8. Brasileiro A, Campos S, Cabete J, Galhardas C, Lencastre A, Serrão V. Trichoscopy as an additional tool for the differential diagnosis of tinea capitis: a prospective clinical study. Br J Dermatol. 2016;175(1):208-9.

9. Ekiz Ö, Şen BB, Rifaioğlu EN, Balta I. Trichoscopy in paediatric patients with tinea capitis: A useful method to differentiate from alopecia areata. J Eur Acad Dermatology Venereol. 2014;28(9): 1255-8.

10. Sastroasmoro S, Ismael S. Dasar-Dasar metodologi penelitian klinis. 5th ed. Jakarta: Sangung Seto; 2014.

11. Hernández-Bel P, Malvehy J, Crocker A, Sánchez-Carazo JL, Febrer I, Alegre V. Comma Hairs: A New Dermoscopic Marker for Tinea Capitis. Actas DermoSifiliográficas.2012;103(9):836-7.

12. Hughes R, Chiaverini C, Bahadoran P, Lacour J-P. Corkscrew Hair: A New Dermoscopic Sign for Diagnosis of Tinea Capitis in Black Children. Arch Dermatol. 2011;147(3):355. 